\title{
Discussing the Effects of Poor Health Literacy on Patients Facing HIV: A Narrative Literature Review
}

\author{
Rocco Palumbo*
}

\begin{abstract}
Background: Scholars describe poor health literacy as a "silent epidemic," which is challenging the functioning of healthcare systems all over the world. Health literacy is mainly meant as an individual trait which concerns the ability to obtain, process, and understand basic health information in order to effectively navigate the health system. Low health literate patients perceive poor self-efficacy dealing with their health conditions, are not willing to be involved in the provision of care, show larger risks of hospitalization and mortality, and are not aware about the determinants of well-being. Hence, limited health literacy has been associated with inadequate management of long-term conditions; nonetheless, several authors argue that health literacy has been an overlooked factor dealing with HIV.

Methods: This study is aimed at discussing the effects of poor health literacy on people living with HIV, drawing from the findings of a narrative literature review which involved 41 papers retrieved from the databases "Scopus-Elsevier" and "PubMed."

Results: The scientific literature is not consistent dealing with the relationship between health literacy and HIV treatment. For example, health literate patients seem to better understand their health conditions; on the other hand, people living with poor health literacy are likely to report higher compliance with providers' prescriptions, blindly trusting healthcare professionals.

Conclusions: Poor health literacy is a social barrier to access healthcare services and to appropriate health treatment among patients living with HIV. Tailored interventions should be aimed at enhancing the health skills of patients affected by HIV infection to improve their ability to navigate the health system.

Keywords: Health Literacy, HIV, Health Promotion, Medication Adherence, Health Education

Copyright: $\odot 2015$ by Kerman University of Medical Sciences

Citation: Palumbo R. Discussing the effects of poor health literacy on patients facing HIV: a narrative literature review. Int J Health Policy Manag. 2015;4(7):417-430. doi:10.15171/ijhpm.2015.95
\end{abstract}

\section{Article History:}

Received: 30 January 2015 Accepted: 9 May 2015 ePublished: 13 May 2015

View Video Summary 0

\section{*Correspondence to:} Rocco Palumbo

Email: rpalumbo@unisa.it

\section{Background}

Simonds ${ }^{1}$ formerly introduced the "health literacy" concept, maintaining that health education is a crucial policy issue to improve the effectiveness of healthcare and bridge health inequalities. $^{2,3}$ In fact, health education deeply affects the functioning of the healthcare system. In particular, it encourages patient involvement in the provision of care, thus enhancing both the appropriateness and equity of services provided. In the light of its derivation from the education field, health literacy was originally conceptualized in a narrow way, in terms of "functional health literacy." Indeed, it was understood as the "ability to use written and oral material to function in healthcare settings." According to this interpretation, health literacy involves the ability to handle the different kinds of data and information which concern the management of the health conditions, thus paving the way for an enhanced proficiency to navigate the health system. ${ }^{6,7}$ Therefore, both the ability to comprehend written and verbal health information (literacy) and the ability to process the numerical data incorporated in the latter (numeracy) are included among the basic skills which build functional health literacy. ${ }^{8,9}$

Alternatively, Nutbeam ${ }^{10}$ brings up the evolving nature of the health literacy concept, suggesting that it consists of several competencies which go beyond functional ones, including interactive and critical skills. On the one hand, patients should establish clear and comfortable relationships with healthcare providers, in order to improve their ability to collect and process relevant health information. ${ }^{11}$ On the other hand, they should develop the ability to explore the alternative treatments available, in order to select the best options to protect and promote their health status. ${ }^{12}$

Merging these two interpretations and drawing from the definitions arranged by the Institute of Medicine ${ }^{13}$ and the United States Department of Health and Human Services, ${ }^{14}$ health literacy could be ultimately described as "the degree to which individuals have the capacity to obtain, process, and understand basic health information and services needed to make appropriate health decisions." 15 Sticking to this definition, most of the scholars and practitioners have dealt with health literacy as an individual trait, ${ }^{16-19}$ claiming that the inadequate understanding of health information is a mainly personal fault of the patient.

In line with this assumption, Parker and colleagues ${ }^{20}$ forecasted a trend of inadequate health literacy for the near future. In fact, the ageing of the population and the correlated epidemiological transition from the prevalence of acute conditions to the preponderance of chronic diseases are supposed to generate poorer ability to face increasingly complex health issues. ${ }^{21}$ Besides, the increased availability of 
health information acts on the patients' information-seeking behavior, requiring enhanced critical skills to discern the utility of accessible data. ${ }^{22}$

\section{The Effects of Health Literacy on Medication Adherence}

Health information is usually complex and ambiguous, especially for patients who show limited health literacy. The uncertainty which is associated with health information challenges the individual ability to make informed choices and to effectively participate in the process of healthcare provision. ${ }^{23}$ In turn, the ability of patients to understand health information and to properly access healthcare services relies on their involvement. ${ }^{24,25}$ From this point of view, limited health literacy produces a vicious cycle, which engenders professional dominance and patient disengagement. ${ }^{26}$ Actually, patients living with limited health literacy are likely to: exhibit impaired ability to recall health information, ${ }^{27}$ perceive low self-efficacy in understanding and using prescription medication, ${ }^{28}$ be unable to devise appropriate strategies to improve medication usage, ${ }^{29}$ reveal health exacerbation, ${ }^{30}$ show repeated and serious medication errors, and cause high medical expenses. ${ }^{31}$

Several studies have discussed health literacy as a significant barrier to medication adherence, ${ }^{32,33}$ although other scholars support that the relationship between these two dimensions is not clear. ${ }^{34}$ In particular, health literacy is assumed to affect a lot of factors which are crucial to treatment adherence, such as: the awareness of the potential uses of medications; the understanding of the modality of drugs' absorption; the consciousness of both beneficial and harmful effects which could be ascribed to medications; the sensitivity of drugs' interactions and side effects; and the perception of the reliability of the information sources which demonstrate medication effectiveness and appropriateness. ${ }^{35}$ Therefore, several authors have claimed that limited health literacy produces inadequate knowledge of medication efficacy, poor self-management of care, low health outcomes, as well as unwillingness to ask both healthcare professionals and informal caregivers for help in managing health conditions. ${ }^{36,37}$ Dealing with postdischarge medication adherence, Lindquist and colleagues ${ }^{38}$ emphasized that health literacy and medication discrepancies were strongly related. In fact, patients with inadequate and marginal health literacy were found to have unintentional nonadherence due to the misunderstanding of health information. On the other hand, health literate patients were more likely to exhibit intentional nonadherence, due to their propensity to deliberately depart from clinical prescriptions. Patients with limited health literacy are also supposed to be unable to accurately report their preadmission medications, thus displaying high risks of inappropriateness. ${ }^{39}$ Besides, health literacy is associated with disease state control, which in turn leads to awareness of health conditions and to treatment adherence. ${ }^{40}$ In sum, patients with low health literacy are more likely to show poorer health conditions, deficient understanding of the determinants of health status, and inadequate ability to navigate the health system as compared with their health literate counterparts.

However, the findings of the scientific literature about the relationship between health literacy and medication adherence are not consistent. For the sake of the argument, dealing with the self-management of diabetes, Rodrigue ${ }^{41}$ maintained that low health literate patients were not able to take part in clinical decision-making and were poor in adhering to treatment regimens. Similarly, Inoue and colleagues $^{42}$ observed that health literacy skills are essential to understand diabetes and participate in the provision of care. Alternatively, two recent literature reviews performed by Fransen and colleagues ${ }^{43}$ and by Loke and colleagues ${ }^{44}$ have found inadequate evidences for a statistical association between health literacy and medication adherence, as well as between health literacy and diabetes self-management, thus claiming that further developments are imperative to shed light on this crucial issue.

The same ambiguity could be detected in the studies discussing the relationship between health literacy and medication adherence of people living with HIV. Among the others, Kalichman and colleagues ${ }^{45}$ argued that poor health literate patients encounter several barriers to medication adherence and that they are not able to selfmanage their health conditions when the support of healthcare professionals and informal caregivers is lacking. Indeed, several scholars claimed that specific interventions tailored to the individual levels of health literacy should be devised to improve medication adherence of people with limited health literacy skills. ${ }^{46}$ On the contrary, Colbert and colleagues $^{47}$ suggested that perceived self-efficacy deeply affects medication adherence, while functional health literacy is not related to either medication adherence or self-efficacy beliefs. Moreover, a recent study about adolescents living with HIV infection showed that health literacy affected neither medication adherence nor self-efficacy perception, even after controlling for age and education levels. ${ }^{48}$

\section{The Consequences of Poor Health Literacy on the Treatment of HIV}

Even though the scientific literature is not unanimous in discussing the impacts which are associated with limited health literacy, ${ }^{49}$ many scholars maintain that poor health literate patients: (1) perceive low self-efficacy dealing with their health conditions; (2) are not willing to be involved in the provision of care; (3) show larger risks of hospitalization; and (4) are not aware of the determinants of well-being. ${ }^{50,51}$ In the light of these insights, both scholars and practitioners have presented inadequate health literacy as a "silent epidemic"20,52,53 which is affecting most of the worldwide population. ${ }^{54-56}$

Claiming that patients have an important role to play when they deal with the protection and the promotion of their health status, ${ }^{57}$ several authors have examined how health literacy affects the management of long-term conditions..$^{58,59}$ Indeed, since adequate health literacy improves the ability to use health information, it incites patient empowerment ${ }^{60}$ and participates in removing the cultural barriers to selfcare. ${ }^{61}$ Moreover, health literacy enhances the exchange of information between the providers and the recipients of care, thus facilitating both shared clinical decision-making and patient involvement. ${ }^{62}$ However, in spite of its relevance to the management of long-term conditions, health literacy is still an overlooked factor when dealing with HIV treatment. ${ }^{63}$ 
Even though studies aimed at discussing the relationship between health literacy and the appropriate treatment of HIV infection are increasingly gaining importance among both scholars and practitioners, the association between these two dimensions is still poorly understood.

This study is aimed at shedding light on this critical issue, drawing from the findings of a narrative literature review which included 41 scientific articles collected from the databases "Scopus-Elsevier" and "PubMed." Rather than systematizing the scientific literature, this paper intends to provide several exploratory insights about the association between health literacy and HIV treatment, in order to incite further conceptual and empirical developments. The article is organized as follows: the next section describes in detail the methodology of the research, depicting both the research process and the eligibility and admissibility criteria for inclusion in the literature review. The third and fourth sections respectively include the examination and the discussion of the findings. Last but not least, the concluding section includes theoretical and practical implications, which turn out to be beneficial to both scholars and practitioners.

\section{Methods}

According to the prevailing scholarship, ${ }^{64,65}$ a clear search and selection strategy is crucial to the success of a literature review. In fact, this research method is aimed at providing a thorough and critical analysis of the relevant and available scientific literature about the topic being studied. In particular, for the purpose of this paper a compound query was performed on the databases "Scopus-Elsevier" and "PubMed." The former represents the largest abstract and citation database of peer-reviewed literature in the fields of science, technology, medicine, social sciences, and arts and humanities. The latter is the widest source of citations for biomedical literature. Other sources were not contemplated, due to their poorer coverage compared with "Scopus-Elsevier" and their wider focus compared with "PubMed." Actually, the breadth and the depth of coverage allowed by these two databases are consistent with both the multidisciplinary nature of the health literacy concept and the specific purposes of this research. Since the primary concern of this study was to examine the effects of health literacy on the health status of patients living with HIV infection, the compound term "health literacy" was employed as the principal key of research to retrieve the relevant literature. This research criterion was typed on the field "Article Title" of the main search interface of the databases consulted. It was associated with a secondary key of research - "HIV" - through the Boolean operator "AND." The secondary research criterion was run on the search field "Article Title, Abstract, Keywords." This asymmetric search strategy was aimed at collecting the scientific literature which showed a strong concern toward health literacy - as shown by the inclusion of this concept in the title of the paper - and, at the same time, discussed the effects of limited health literacy on the health conditions of patients living with HIV infections. The Author did not introduce any temporal limitation, in order to avoid the exclusion of potentially significant articles. Besides, all the subject areas listed by "Scopus-Elsevier" and "PubMed" were contemplated, including Life Sciences, Physical Sciences, Health Sciences, and Social Sciences.
For the sake of both efficiency and robustness, the attention was focused on articles and reviews published on peerreviewed journals. Otherwise, other contributions-such as editorials, commentaries, and letters to the editor-were ignored. Similarly, grey literature, books and book chapters were not considered, in order to enhance the reliability of this study by involving only contributions published in highquality peer-reviewed sources. Both conceptual and empirical papers were included in the research to catch all the shades of the topic being examined. The last query was run on January 7, 2015 on both the citation databases.

As shown in Figure 1, 95 papers published between 1999 and 2014 were collected through this research process. Fortythree records appeared in the results of both the databases and were consequently removed, since redundant. In addition, 6 papers were not included in the analysis, because their abstracts were not available. Consequently, 46 papers were taken into consideration. After screening their titles and abstracts, 5 of them were recognized as not relevant for the purpose of this study, since they did not adequately address the impacts of health literacy on the treatment of HIV conditions. The full-text of the remaining 41 articles was acquired from either Scopus-Elsevier, PubMed, or external sources. Then, the collected papers were analysed in-depth in order to collect evidence of the effects of health literacy on the health conditions of patients living with HIV infection. More than half of the papers ( 22 out of 41 ) were published in the period 2010-2014, pointing out the timeliness of the topic being discussed. In line with this assumption, only 7 out of 41 papers were published before 2004 .

Most of the papers (about $70 \%$ of the total) belonged to the subject areas "Medicine" and "Nursing." Six out of 41 papers were included in the "Social Science" field, while 5 of them fit in the "Psychology" one. Only one of the retrieved papers was included in the "Pharmacology, Toxicology and Pharmaceutics" area. Three of the collected papers were review articles, 3 were conceptual papers, and the other 35 were original research articles. Most of the retrieved papers concerned the United States, while only a minority of them dealt with the association between health literacy and HIV

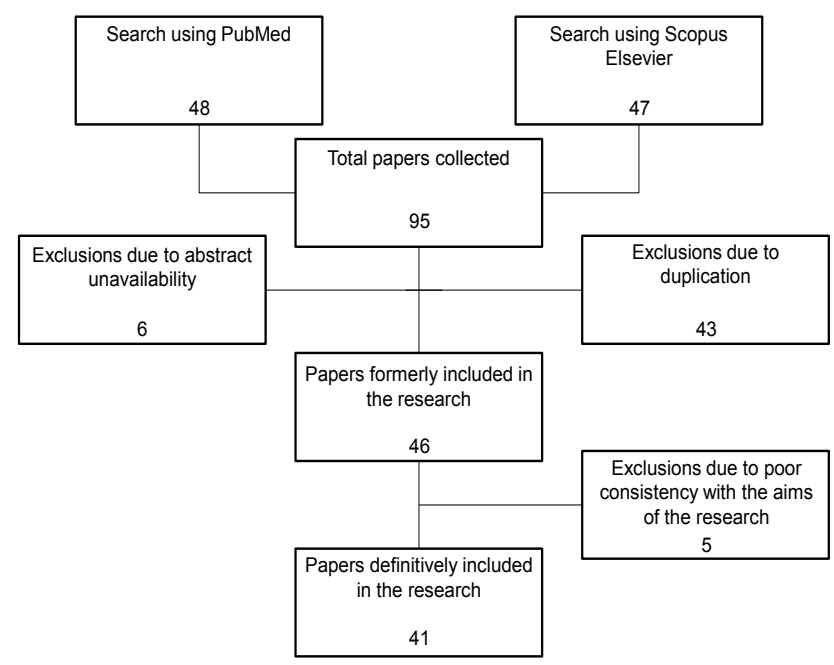

Figure 1. Flowchart of Papers' Search and Selection. Source: Author's elaboration. 
conditions of patients living in other countries, suggesting a strong geographic concentration of the research in this field. Table provides a synthesis of the retrieved papers, depicting their characteristics in terms of research type, health literacy tool employed, study design, sample description, and main findings achieved. Most of the papers included in this literature review presented the results of cross-sectional studies, clinical trials, and pilot studies, which mainly involved nonrepresentative and/or convenience samples. In several cases qualitative methods were adopted, such as focus groups, case studies, and semi-structured interviews involving participants recruited from specific clinic and community-based settings. As a consequence, the findings of most of the papers included in this literature review are not generalizable. Rather, they are indicative of trends specific to a particular sample, providing some food for thought for further developments.

Moreover, the consistency of the results depicted in this narrative review is affected by the variety of the instruments administered to participants to assess their level of health literacy. The Test of Functional Health Literacy in Adults (TOFHLA) and the Rapid Estimate of Adult Literacy in Medicine (REALM) are the most common tools used to assess individual health literacy. The former measures the patients' ability to read and understand basic health-related prose messages and numerical information, while the latter is a word-recognition test aimed at predicting general reading ability. In both cases, poor attention is paid to the individual ability to process and understand specific health information which is crucial to manage HIV infection. Only in few cases the original version of either the TOFHLA or the REALM were adapted to consider the information needs of patient affected by HIV infection. As well, tailored tools to measure the health literacy levels of patients living with HIV were infrequently used, including the Brief Estimate of Health Knowledge and Action-HIV (BEHKA-HIV) version, the HIV-Related Health Literacy Scale, and the HIV Literacy Test.

\section{Results}

Health Literacy as a "Social Barrier" to Access Healthcare Services

Inadequate health literacy has been usually described as a strong barrier to access healthcare services. Moreover, it is assumed to affect medication adherence and compliance with medical prescriptions. Indeed, in a recent study aimed at examining the antiretroviral medication adherence of poor health literate individuals living with HIV, limited health literacy was found to be associated with greater illness severity and weak health conditions. ${ }^{66}$

Kalichman and Rompa ${ }^{67}$ formerly supported that health literacy performs as a significant predictor of well-being among people living with HIV. In fact, poor health literate patients affected by HIV infection are likely to demonstrate difficulty comprehending simple medical instructions and to access healthcare services. Besides, they have been recently found to show deficient knowledge of their HIV-related health status, ${ }^{68}$ low compliance with clinical prescription, ${ }^{66}$ inadequate treatment awareness, ${ }^{69}$ and poor medication adherence. $^{70}$ From this point of view, it is argued that people living with HIV and limited health literacy skills are expected to report lower health conditions as well as greater number of hospitalizations compared to their health literate counterparts. ${ }^{67}$ This assumption is of particular interest, since the hospital setting paves the way for increased risks of inappropriateness dealing with long term conditions in general, and HIV infection in particular.

Analogous findings are discussed by Kalichman and colleagues, ${ }^{71}$ who, drawing from the results of a cross-sectional study examining health literacy in relation to knowledge of HIV, claimed that limited health literacy prevents full understanding of health conditions, illness, and treatments. Besides, poor health literate patients are more likely to exhibit misinterpretation of the expected outcomes of HIV treatments. To make things worse, inadequate medication adherence and dosing errors were observed among adult informal caregivers with marginal health literacy skills. ${ }^{72}$ Since people living with HIV are likely to show strong reliance on the support and counselling of informal caregivers, this finding is crucial, even though still little is known about this issue among both scholars and practitioners. On the one hand, the scientific literature seems to overlook the effects of informal caregivers' limited health literacy on the health status of their cared-for, mainly focusing on specific concerns, such as caregivers' adherence to pediatric antiretroviral medications. On the other hand, the information needs of informal caregivers living with poor health literacy are widely neglected by healthcare providers, thus undermining their ability to properly navigate the healthcare system.

As well, stigma is a common and critical problem among poor health literate patients who face concealable conditions, such as HIV. In fact, as argued by Zukoski and colleagues, ${ }^{73}$ stigma is associated with feelings of anger, fear, and concern about disclosure, which turn out to be significant barriers to access healthcare services. Therefore, it contributes to adverse health consequences and to the exacerbation of health conditions. ${ }^{74}$ In addition, stigma produced by poor health literacy compounds stigma related to being HIV-affected, exacerbating the overall negative effects on the individual ability to follow appropriate health treatments. In fact, the fear of stigma and discrimination prevents the establishment of a clear and comfortable relationship between the patients and the providers of care, frustrating the ability of the former to be involved in the provision of care and the willingness of the latter to implement patient-centered communication approaches.

In sum, as shown in Figure 2, inadequate health literacy impoverishes the ability to find and use relevant health information. Actually, limited health literacy could be understood as a predictor of: poor understanding of health information, misinterpretation of expected outcomes of health treatments, impaired awareness of the consequences of HIV condition, and stigma. In turn, these factors operate as significant barriers to access healthcare services. As a consequence, they prevent the appropriate treatment of HIV infection and hinder the achievement of substantial health improvements.

The Drawbacks of Poor Health Literacy on HIV Treatment The prevailing literature supports that health literacy is an independent determinant of medication nonadherence. 


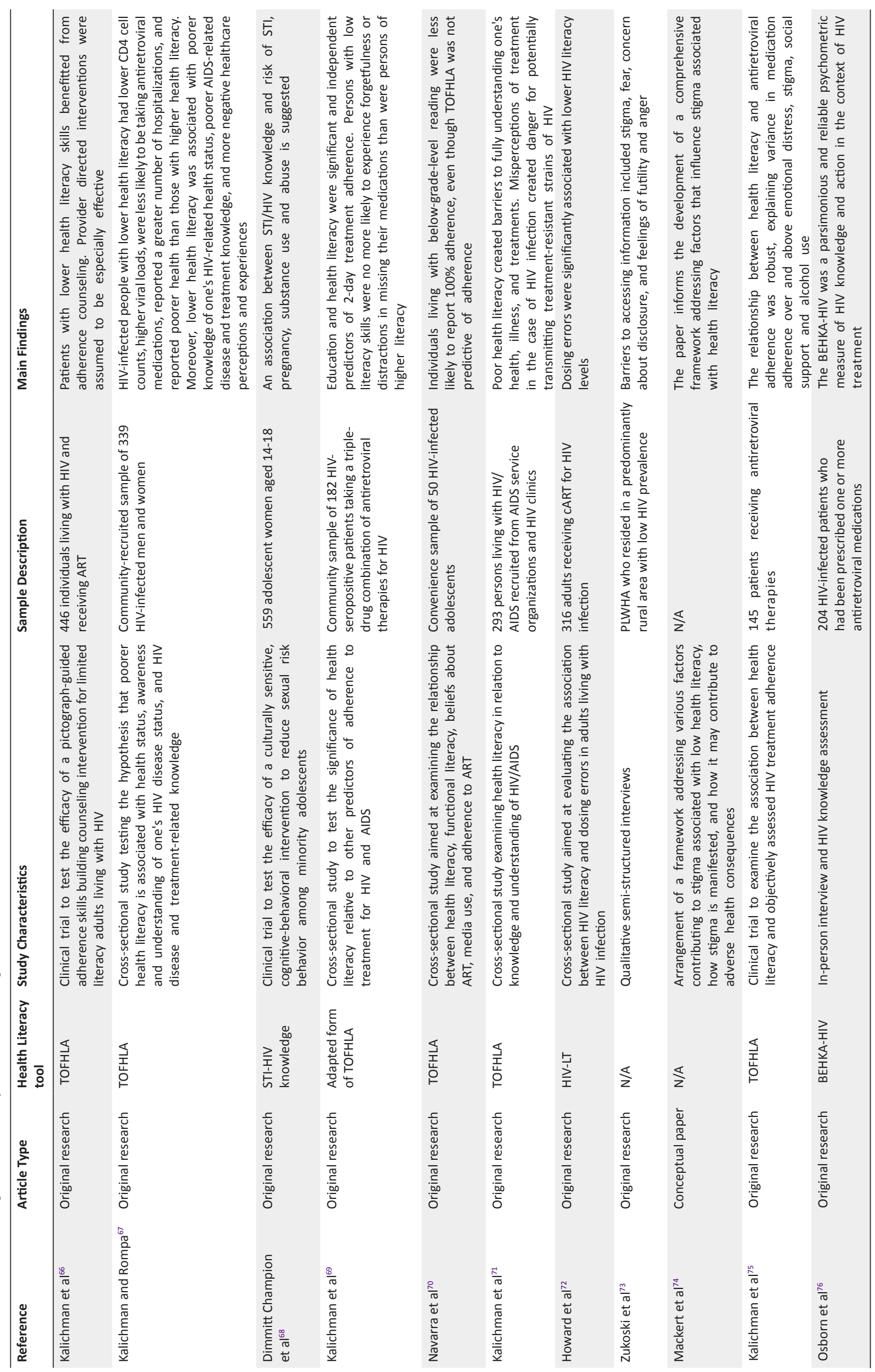




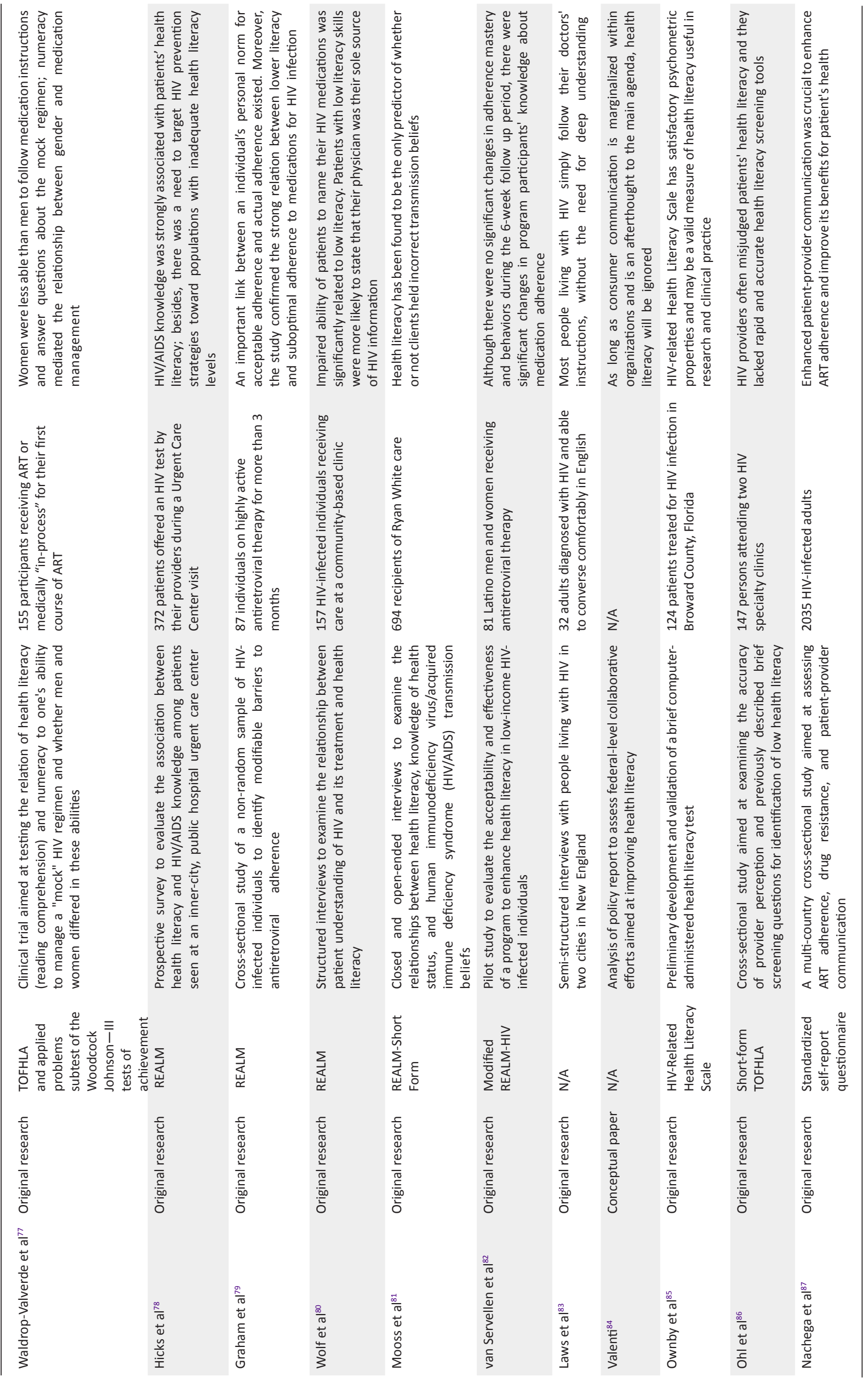




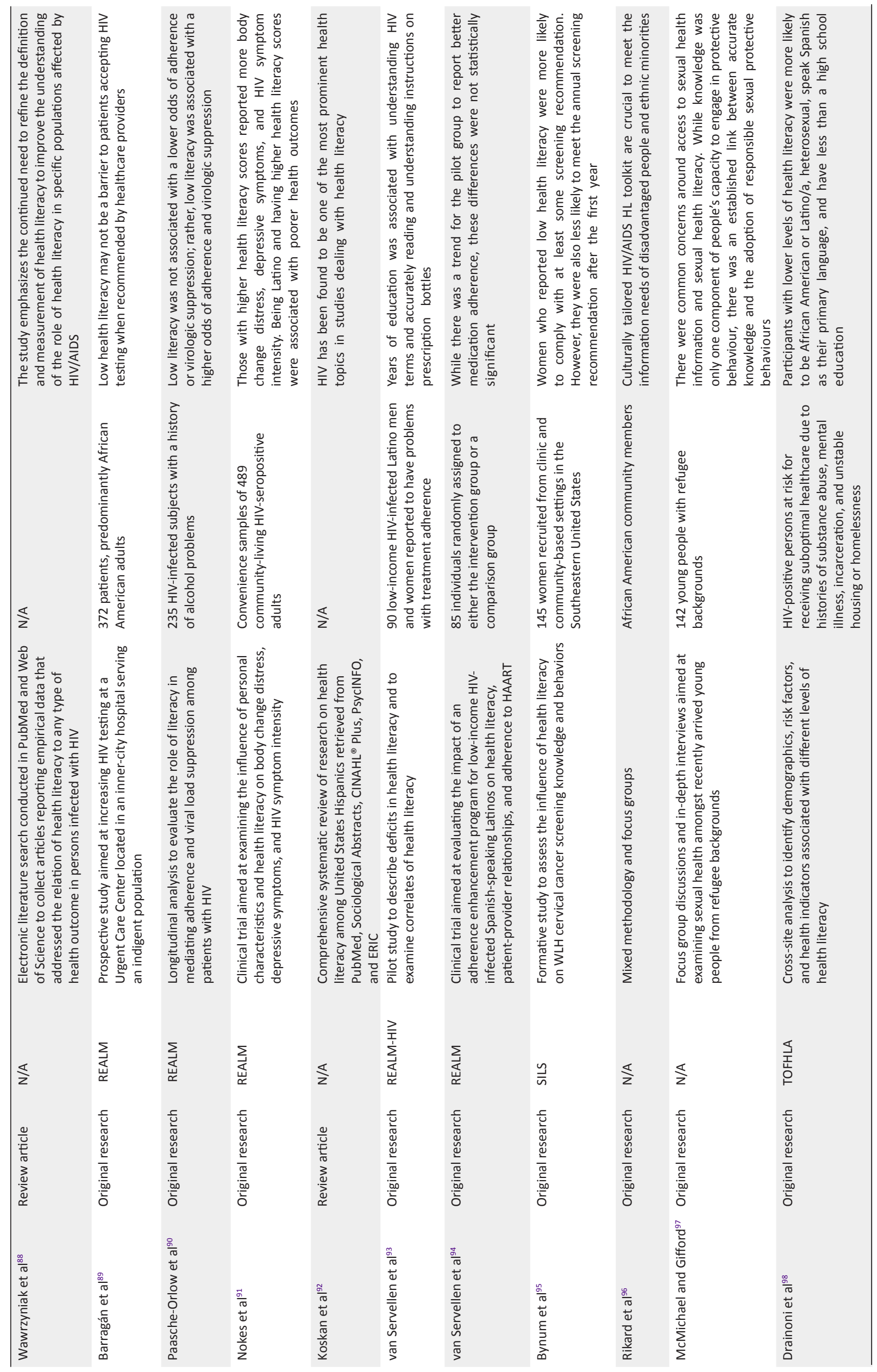




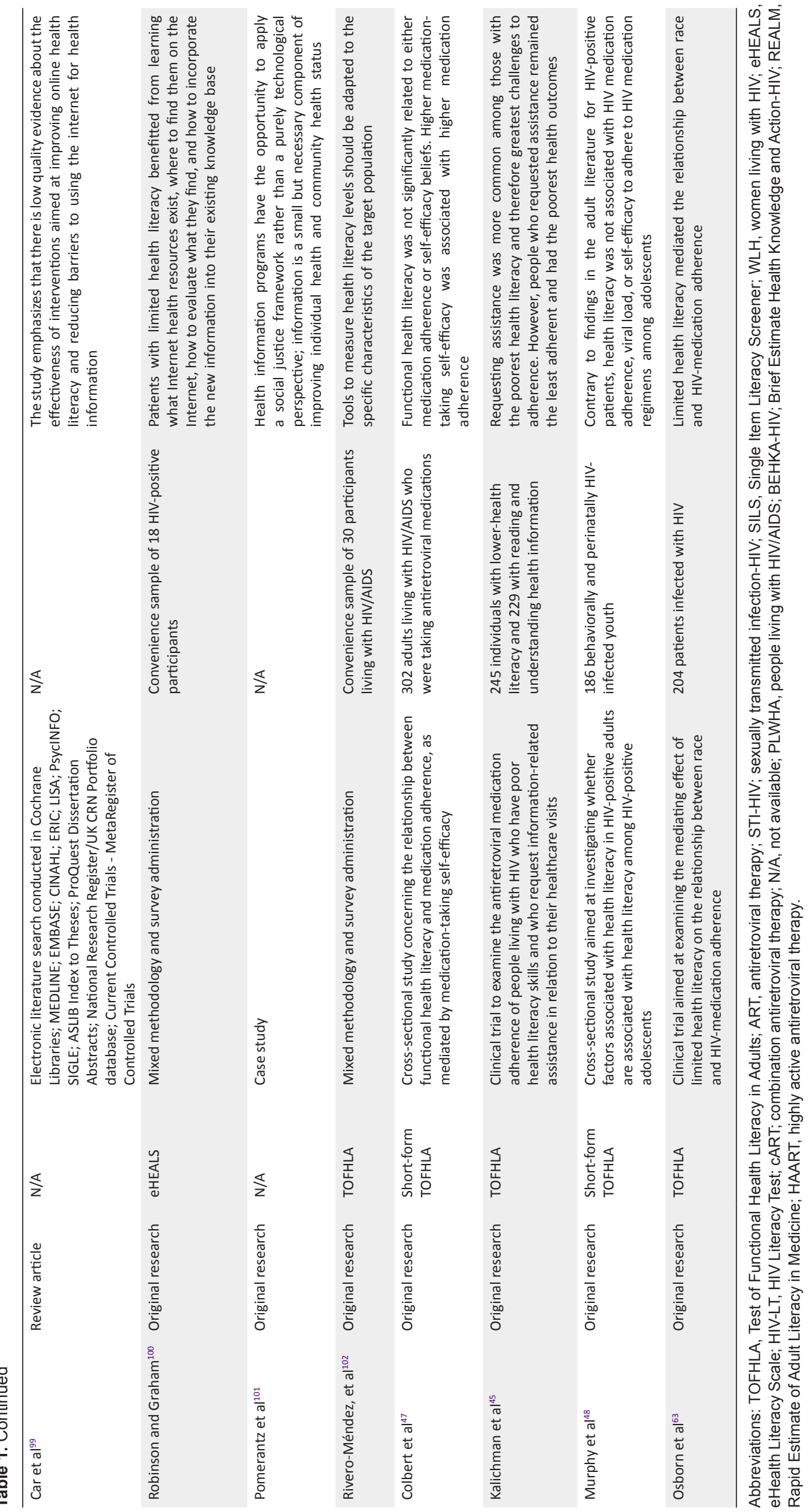




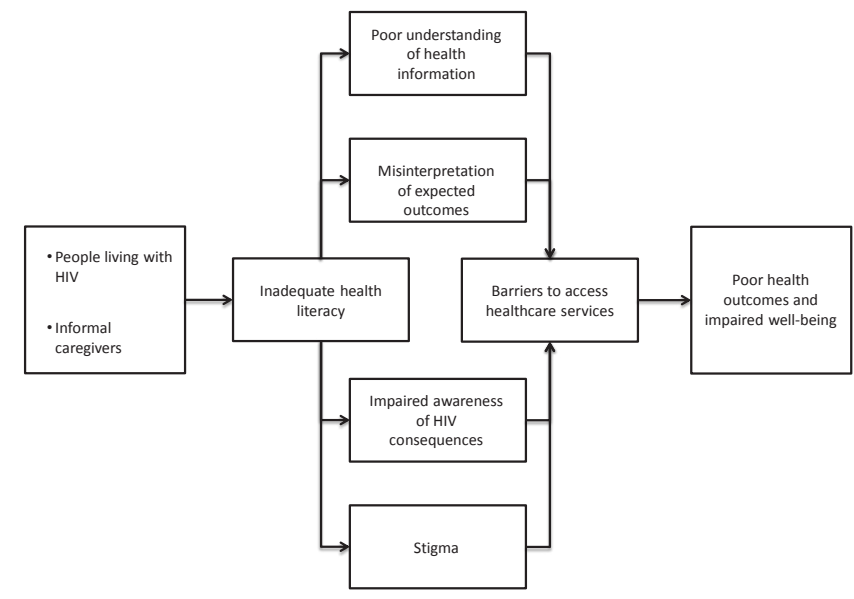

Figure 2. Inadequate Health Literacy as a Social Barrier to Access Healthcare. Source: Author's elaboration.

In fact, a cross-sectional study aimed at examining the association between health literacy and objectively assessed HIV treatment adherence found the relation between these two dimensions to be robust, with health literacy predicting adherence over and above all other factors. ${ }^{75}$ As well, through multivariate regression models examining the associations among race, literacy, and HIV-medication adherence in a convenience sample of HIV infected patients recruited from two different clinics, Osborn and colleagues ${ }^{76}$ found that health literacy mediated the relationship between race and poor HIV-medication adherence, performing as a significant independent predictor of nonadherence. Waldrop-Valverde et $\mathrm{al}^{77}$ expanded these findings, suggesting that health literacy skills - especially functional ones - mediated the impacts of gender on the appropriate medication management of HIV. From this point of view, it could be argued that health literacy plays an important role in attenuating social disparities in the access to care and enhancing the proper treatment of HIV-related conditions. Drawing from these assumptions, in a prospective survey to evaluate the association between health literacy and HIV/AIDS knowledge Hicks and colleagues $^{78}$ identified a significant relation between these two dimensions, which is not mediated by income, education, and risk perception. Obviously, a better knowledge of HIV-related conditions paves the way for a more appropriate management of the health status, as well as for increased health outcomes. In line with these results, health literacy is supposed to be a significant and independent predictor of treatment adherence, even after adjusting for age, ethnicity, and social support. ${ }^{69}$ Moreover, the association between limited health literacy and poor treatment adherence has been found to be unaltered by mistaken beliefs regarding taking HIV medications. ${ }^{79}$ Similarly, marginal and inadequate health literacy have been related to the inability to name HIV medications and to the impaired awareness of medication usage rather than to perceived self-efficacy. ${ }^{80}$ Last but not least, poor health literate people are believed to exhibit low knowledge of sexually transmitted HIV infection, thus being unable to prevent its transmission. ${ }^{68}$

Limited health literacy was also found to: produce the inability to control the health status, ${ }^{67}$ hamper the establishment of positive and comfortable relationships with healthcare providers, ${ }^{81}$ and foster involuntary disengagement with the prescribed treatment. ${ }^{69}$ Moreover, the side effects of health literacy on HIV treatment are amplified by disadvantaged social conditions. In fact, the underserved population - such as rural communities and racial minorities - are assumed to be at greater risk of inadequate health literacy and inappropriate management of HIV-related conditions. ${ }^{82}$

\section{The "Dark Side" of Health Literacy}

Quite surprisingly, several authors have argued that health literacy is not strictly required for good medication adherence of people living with HIV, claiming that most of them simply follow the providers' instructions, without the need for understanding health information. ${ }^{83}$ Besides, in spite of what has been discussed above, data and evidence linking health literacy to outcomes in HIV care are limited. ${ }^{84}$ In particular, the traditional measures of health literacy - the TOFHLA and the REALM among the others - show several content and format limitations, which make them not particularly fitting with the specific challenges of HIV treatment. ${ }^{85,86}$ Nevertheless, they continue to be used in most of the studies examining the effects of health literacy on health outcomes in people living with HIV. As a consequence, still little is known about the relations linking, on the one hand, health literacy with HIV treatment adherence ${ }^{87}$ and, on the other hand, health literacy with the health status of patients affected by HIV infection. ${ }^{88}$

Indeed, several scholars maintain that functional health literacy is not significantly related to medication adherence and appropriate treatment of HIV-related conditions. For example, in their cross-sectional study Colbert et $\mathrm{al}^{47}$ claimed that health literacy was neither associated with medication adherence nor with self-efficacy beliefs. In turn, self-efficacy beliefs were assumed to positively affect patients' compliance with clinical prescriptions. In a similar way, health literacy was found to be related neither to medication adherence nor to self-efficacy perception of adolescents living with HIVinfection, even though it was correlated with the amount of medical care received. ${ }^{48}$ These findings are of particular interest: on the one hand, they point out that HIV-infected adolescents have special information needs in comparison with other categories of patients, since they are likely to rely upon the support of informal caregivers when dealing with healthcare providers. On the other hand, the health literacy skills of informal caregivers are expected to deeply affect medication adherence and appropriateness of care of young persons living with HIV infection.

Barragán and colleagues ${ }^{89}$ discussed analogous findings, suggesting that neither education nor health literacy is able to affect acceptance or refusal of HIV testing in the healthcare setting. Actually, low health literate patients were found to be more likely to accept HIV testing and to be more willing to comply with the recommendations of healthcare professionals as compared with health literate individuals. In addition, Paasche-Orlow et $\mathrm{al}^{90}$ supported that people with the lowest literacy levels were likely to exhibit higher odds of medication adherence and HIV-RNA suppression in comparison with health literate ones, even after adjusting for confounding factors. In the light of these results, the authors proposed that poor health literate patients face significant 
difficulties when they have to acquire the self-management skills required for a new drug regimen. On the other hand, they are able to follow medical prescriptions more readily once in a therapeutic steady state as compared with their health literate counterparts.

Nokes and colleagues ${ }^{91}$ argued that these counter-intuitive findings challenge the utility of the traditional tools to measure health literacy with regard to the management of HIV-related conditions. As well, supporting that robust longitudinal studies are needed to grasp the complex relationships between health literacy and health outcomes in persons living with HIV, they provide some food for thought which incites further developments to expand both the scientific and practical knowledge about the effects of health literacy on the proper treatment of HIV-related conditions. Specifically, greater attention should be paid to the confounding factors which affect the relationship between health literacy and the management of HIV infection, including - as an illustrative example - the predisposition of patients to voluntarily depart form clinical prescriptions and their willingness to follow alternative and unconventional health treatments.

The Promotion of Health Literacy Skills Among People Living With HIV

Although the findings of the scientific literature are not consistent, HIV infection continues to be among the most prominent health topics addressed by the scholars dealing with health literacy. ${ }^{92}$ On the one hand, health literacy has been identified as the only one significant predictor of incorrect transmission beliefs among people living with HIV. ${ }^{81}$ On the other hand, patients with the lowest levels of health literacy are more likely to need informational assistance and external support to improve their adherence strategies. ${ }^{66}$

From this point of view, adherence enhancement programs targeted to the specific characteristics of the least literate groups of people living with HIV are imperative to improve both medication adherence and health outcomes. ${ }^{93,94}$ Since healthcare professionals are usually perceived by patients with limited health literacy as the most trusted and reliable source of health information, provider-directed interventions are considered to be especially effective. ${ }^{66}$ The same is true with regard to HIV-related illnesses, such as cervical cancer. ${ }^{95}$ Therefore, the enhancement of health literacy is understood as a crucial strategy to improve the health, social, and economic outcomes of interventions aimed at addressing long-term conditions, including HIV. ${ }^{103}$ From this point of view, in spite of the prevailing disagreement among scholars, health literacy is meant as an important determinant of healthy life styles and health status, especially among underserved ethnic minority groups, ${ }^{93,97}$ people with refugee backgrounds, ${ }^{98}$ and individuals at risk of receiving suboptimal and/or inappropriate health treatments due to histories of substance abuse, mental illness, incarceration, and unstable housing or homelessness. ${ }^{99}$

The increasing availability of health information on the internet poses further challenges for poor health literate individuals living with HIV. In fact, several barriers prevent them from making effective use of these important sources of information, such as inadequate informatics skills and poor ability to search, evaluate and use health information. ${ }^{100}$
Consequently, Robinson and Graham ${ }^{101}$ argued that introductory internet health information educational interventions tailored to patient affected by HIV are crucial to improve their confidence in finding and using health information resources, since they enhance the individual ability to self-manage the health condition.

At the same time, community-based health information partnerships seem to be particularly effective in addressing health literacy and health information inequalities in marginalized communities. In fact, they act as learning environments for people with marginal health literacy skills, concurring to enhance their ability to collect, process, and understand health information. ${ }^{102}$ Obviously, both health literacy measures and interventions aimed at fostering the individual ability to handle health data should be tailored to the specific social and epidemiological characteristics of patients diagnosed with HIV infection, in order to achieve the highest levels of effectiveness. ${ }^{103}$

\section{Discussion}

The role played by health literacy in the adequate treatment of HIV infection is a timely and interdisciplinary topic, which concerns different subject areas, including medicine, nursing, social sciences, psychology, and pharmacology. In spite of its relevance, the scientific research on this issue shows a significant geographic concentration, being mainly contributed by the United States. Nonetheless, several streams of research are increasingly developing in other areas of the world, especially in Europe and Australia. This phenomenon indicates a slow raising of the consciousness at the international level about the importance of health literacy in the appropriate management of HIV-related conditions. The effects of health literacy on the health conditions of people living with HIV infection are still poorly understood. Actually, data and evidence discussed by both the scientific and the professional literatures are limited and - in several cases - conflicting. Such an ambiguity encourages further developments aimed at shedding light on this critical issue. Inadequate health literacy has been usually depicted as a social barrier to access healthcare, paving the way for greater illness severity and impaired health conditions. In fact, low health literate patients living with HIV infection are expected to show deficient knowledge of their health status, which in turn produces low compliance with clinical prescriptions, inadequate treatment awareness, and poor medication adherence. Moreover, poor health literate patients are likely to demonstrate difficulty comprehending health information, to develop exacerbation of their health condition, and to show greater number of hospitalization as compared with their health literate counterparts.

To make things worse, inadequate health literacy concurs in deepening the stigma which is usually attached to concealable health conditions, such as HIV. As a consequence, limited health literacy engenders a biased and uncomfortable relationship between the patients and the providers of care. The mix of HIV related conditions and inadequate health literacy produces anger, fear, and concern about disclosure, which furthermore prevent the appropriate access to healthcare and discourage the patient engagement in the management of healthcare. Problems associated with 
health literacy also affect informal caregivers, engendering significant risk of misinterpretation of the expected outcomes of HIV treatments, dosing errors, and overall poor medication adherence.

However, scholars have discussed several counter-intuitive findings, claiming that neither health literacy nor education is able to affect medication adherence and compliance with providers' prescriptions among people living with HIV. In fact, although inadequate health literacy has been generally associated with unintentional nonadherence, some studies have shown that people with inadequate and/or marginal health literacy are more likely to properly follow clinical prescriptions, without the need for understanding. Actually, poor health literate patients seem to be more willing to ask healthcare professionals for help in managing their health conditions in comparison with their health literate counterparts. On the other hand, it could be argued that limited health literacy hinders the self-management of care, thus provoking an excessive dependence of the patient on the providers of care. In these terms, poor health literacy could pave the way for professional dominance and marginal involvement of the patient in the provision of care. This "dark side" of health literacy is still widely unexplored, as also demonstrated by the lack of consistency in the findings of this literature review. As well, the ability of the healthcare system and healthcare organizations to meet the need of low health literate patients living with HIV is generally neglected, as shown by the lack of studies discussing the impacts of organizational health literacy on the enhancement of health outcomes produced by HIV treatments. Hence, further developments could be aimed at examining the way the healthcare system itself prevents the appropriate understanding of health information of poor health literate patients living with HIV.

Several limitations challenge the reliability of this study. First of all, the focus on the databases "Scopus-Elsevier" and "PubMed" met the criterion of efficiency, but it prevented the consideration of other significant sources of scientific articles, which could have been relevant to the purpose of this research. Another weakness could be identified in the search strategy devised to select and collect the articles included in this study. On the one hand, it allowed to consider only the articles whose main concern was health literacy; on the other hand, it caused the exclusion of articles that did not contain the concept "health literacy" in their title, but nonetheless discussed the effects of the latter on the appropriate treatment of HIV-related conditions. Last but not least, the omission of grey literature restricted the breadth of this literature review, but it did not significantly affect the reliability of its results, since the latter were drawn from high-quality articles published by peer-reviewed journals.

In spite of these limitations, this paper paves the way for further researches, which are aimed at enhancing both the scientific and the practical knowledge about the relationship between health literacy and the adequate treatment of HIV infection. First of all, in order to reduce the biases which affect the studies of the effects of health literacy on the management of HIV infection, a more reliable measure of the health literacy skills needed by people living with HIV should be arranged and statistically tested. Such a measure of health literacy should take into consideration the specific attributes and abilities which are required to properly deal with HIVrelated health conditions, contemplating both functional, critical, and interactive competencies.

Moreover, particular attention should be paid to the role played by health literacy in mitigating social, racial, and gender disparities in healthcare, especially among underserved category of patients - including ethnic minority groups, people with refugee backgrounds, inmates, mentally ill, and homelessness. As well, the impacts of marginal and inadequate health literacy on the willingness of people living with HIV to adopt healthy life-styles which favours an appropriate treatment of HIV is a topic which, in spite of its strong relevance, is still poorly discussed.

Lastly, further developments should be directed at examining the characteristics and the outcomes of the interventions to enhance the health literacy of people living with HIV, in particular at the community level. Among the other issues, the drawbacks of the availability of a greater number of health information on the information-seeking behavior of people with limited health literacy skills should be analysed in-depth, with the purpose of better understanding how it is possible to improve the individual ability to handle and process significant health information.

Since both policy-makers and health managers are poorly aware of the role played by health literacy in the treatment of $\mathrm{HIV}$, greater efforts should be made to foster the consciousness of this issue. Similarly, patients and caregivers facing HIVinfection generally overlook the importance of health literacy to the enhancement of the individual health conditions. For these reasons, institutional initiatives aimed at improving the awareness of the effects of health literacy on the appropriate treatment of HIV-related conditions should not be postponed anymore.

\section{Conclusions}

Health literacy is crucial to the appropriate treatment of HIV infection. Nonetheless, the association linking health literacy and health conditions of people living with HIV is still poorly understood. Indeed, the scientific literature shows several counter-intuitive findings, according to which education and health literacy are assumed not to affect the proper management of HIV-related conditions. Alternatively, scholars are consistent in claiming that poor health literate patients are more likely to exhibit unintentional nonadherence compared to their health literate counterparts, who usually display intentional nonadherence. In fact, the latter may be overconfident about their knowledge of HIV status, thus deliberately departing from clinical prescriptions. On the other hand, the former may lack the skills and the processing abilities to appropriately follow the instructions of healthcare professionals, thus being exposed to higher risks of exacerbations.

Even though the scientific research in this field is increasingly growing, health literacy is a widely overlooked issue dealing with HIV treatment. The lack of tailored tools to measure the health literacy skills that are relevant to conveniently manage HIV-related conditions witnesses a poor attention paid to this topic by both scholars and practitioners. In addition, the geographic concentration of studies discussing the impacts 
of health literacy on the treatment of HIV infection suggests the absence of consciousness of the direct and indirect relationships which connect these two variables in most of the countries of the world.

The findings of this paper seek to raise the awareness of both policy-makers and health managers about the role played by health literacy skills in improving the health outcomes achieved by people living with HIV. The former should include health literacy among their main concerns when devising the policies for the revitalization of national health systems. At the same time, the latter should contemplate the interventions aimed at enhancing the individual levels of health literacy among the initiatives which bring to better health outcomes as well as to higher effectiveness of care.

\section{Ethical issues}

As this is a review article, there was no need to obtain approval of an ethics committee.

\section{Competing interests}

Author declares that he has no competing interests.

\section{Author's contribution}

$\mathrm{RP}$ is the single author of the manuscript.

\section{References}

1. Simonds SK. Health education as social policy. Health Educ Mono. 1974;2(1):1-25.

2. Peerson A, Saunders M. Health literacy revisited: what do we mean and why does it matter? Health Promot Int. 2009;24(3):285296. doi:10.1093/heapro/dap014

3. Tones K. Health literacy: new wine in old bottles? Health Educ Res. 2002;17(3):287-290. doi:10.1093/her/17.3.287

4. Parker RM, Baker D, Williams M, Nurss J. The test of functional health literacy in adults: a new instrument for measuring patients' literacy. J Gen Intern Med. 1995;10:537-541.

5. McCarthy A, Jennifer L. Health literacy policy and strategy: Research report for national adult literacy agency. Dublin:National Adult Literacy Agency;2002.

6. Brach C, Dreyer B, Schyve P, et al. Attributes of a health literate organization. Washington, DC:The National Academies Press;2012.

7. Rudd RE. Health literacy skills of U.S. adults. Am $J$ Health Behav. 2007;31(1):8-18

8. Andrus MR, Roth MT. Health literacy: a review. Pharmacotherapy. 2002;22(3):282-302.

9. Estrada C, Barnes V, Collins C, Byrd JC. Health literacy and numeracy. JAMA 1999;282(6):523-529.

10. Nutbeam D. The evolving concept of health literacy. Soc Sci Med. 2008;67:2072-2078. doi:10.1016/j.socscimed.2008.09.050

11. Watermeyer J, Penn C. "Tell me so I know you understand": Pharmacists' verification of patients' comprehension of antiretroviral dosage instructions in a cross-cultural context. Patient Educ Couns. 2009;75(2):205-213. doi:10.1016/j.pec.2008.09.009

12. Stableford S, Mettger W. Plain Language: A Strategic Response to the Health Literacy Challenge. J Public Health Policy. 2007;8(1):71-93.

13. Institute of Medicine. Health literacy: a prescription to end confusion. Washington, DC:National Academies Press;2004

14. US Department of Health and Human Services. Healthy People 2010. Washington, DC:US Government Printing Office;2000.

15. Baker DW. The Meaning and the Measure of Health Literacy. J Gen Intern Med. 2006;21:878-883. doi:10.1111/j.15251497.2006.00540.x

16. French M, Hernandez LM. Organizational Change To Improve Health Literacy: Workshop Summary. Washington, DC:National
Academies Press;2013.

17. Pleasant A, Cabe J, Martin L, Rikard RV. A Prescription Is Not Enough: Improving Public Health with Health Literacy. Washington, DC:Institute of Medicine;2013.

18. Barrett SE, Puryear JS. Health literacy: improving quality of care in primary care settings. J Health Care Poor Underserved. 2006;17(4):690-697.

19. Mika VS, Kelly PJ, Price MA, Franquiz M, Villarreal R. The ABCs of health literacy. Fam Community Health. 2005;28(4):351-357.

20. Parker RM, Wolf MS, Kirsh I. Preparing for an epidemic of limited health literacy: weathering. J Gen Intern Med. 2008;23(8):12731276. doi:10.1007/s11606-008-0621-1

21. Baker DW, Gazmararian JA, Sudano J, Patterson M. The Association Between Age and Health Literacy Among Elderly Persons. J Gerontol B Psychol Sci Soc Sci. 2000;55(6):368-374.

22. Manafo $E$, Wong $S$. Exploring older adults' health information seeking behaviors. J Nutr Educ Behav. 2012;44(1):85-89. doi:10.1016/j.jneb.2011.05.018

23. Peters E, Hibbard J, Slovic P, Dieckmann N. Numeracy skill and the communication, comprehension, and use of risk-benefit information. Health Aff. 2007;6(3):741-748.

24. Raynor DK. Health literacy. Br Med J 2012;344:e2188. doi:10.1136/bmj.e2188

25. Hibbard JH, Peters E, Dixon A, Tusler M. Consumer competencies and the use of comparative quality information: it isn't just about literacy. Med Care Res Rev. 2007;64(4):379-394.

26. Ishikawa H, Yano E. Patient health literacy and participation in the health care process. Health Expect. 2008;11:113-122. doi:10.1111/j.1369-7625.2008.00497.x

27. McCarthy DM, Waite KR, Curtis LM, Engel KG, Baker DW, Wolf MS. What did the doctor say? Health literacy and recall of medical instructions. Med Care. 2012;50(4):277-282. doi:10.1097/MLR.0b013e318241e8e1

28. Cameron KA, Ross EL, Clayman ML, et al. Measuring patients' self-efficacy in understanding and using prescription medication. Patient Educ Couns. 2010;80(3):372-376. doi:10.1016/j. pec.2010.06.029

29. Kripalani S, Gatti ME, Jacobson TA. Association of age, health literacy, and medication management strategies with cardiovascular medication adherence. Patient Educ Couns. 2010;81(2):177-181. doi:10.1016/j.pec.2010.04.030

30. Noureldin M, Plake KS, Morrow DG, Tu W, Wu J, Murray MD. Effect of health literacy on drug adherence in patients with heart failure. Pharmacother. 2012;32(9):819-826. doi:10.1002/j.18759114.2012.01109.x

31. Ngoh LN. Health literacy: a barrier to pharmacist-patient communication and medication adherence. J Am Pharm Assoc. 2009;49(5):132-149. doi:10.1331/JAPhA.2009.07075

32. Blake SC, McMorris K, Jacobson KL, Gazmararian JA, Kripalani S. A qualitative evaluation of a health literacy intervention to improve medication adherence for underserved pharmacy patients. J Health Care Poor Underserved. 2010;21(2):559-567. doi:10.1353/hpu. 0.0283

33. Murray MD, Wu J, Tu W, Clark DO, Morrow DG, Brater DC. Health literacy predicts medication adherence. Clin Pharmacol Ther. 2004;75:76.

34. Zhang NJ, Terry A, McHorney CA. Impact of health literacy on medication adherence. A systematic review and meta-analysis. Ann Pharmacother. 2014;48(6):741-751. doi: $10.1177 / 1060028014526562$

35. Herxheimer A. Public understanding of drug therapy. $\mathrm{Br} \mathrm{J}$ Clin Pharmacol. 2012;73(6):943-947. doi:10.1111/j.13652125.2012.04243.x

36. Devraj R, Herndon CM, Griffin J. Pain awareness and medication knowledge: a health literacy evaluation. J Pain Palliat Care Pharmacother. 2013;27(1):19-27. doi:10.3109/15360288.2012. 751955

37. Federman $A D$, Wolf $M$, Sofianou $A$, et al. The association of health literacy with illness and medication beliefs among older 
adults with asthma. Patient Educ Couns. 2013;92(2):273-278. doi:10.1016/j.pec.2013.02.013

38. Lindquist LA, Go L, Fleisher J, Jain N, Friesema E, Baker DW. Relationship of health literacy to intentional and unintentional non-adherence of hospital discharge medications. J Gen Intern Med. 2012;27(2):173-178. doi:10.1007/s11606-011-1886-3

39. Marvanova M, Roumie CL, Eden SK, Cawthon C, Schnipper $\mathrm{JL}$, Kripalani S. Health literacy and medication understanding among hospitalized adults. J Hosp Med 2011;6(9):488-493. doi:10.1002/jhm.925

40. Keller DL, Wright J, Pace HA. Impact of health literacy on health outcomes in ambulatory care patients: a systematic review. Ann Pharmacother. 2008;42(9):1272-1281. doi:10.1345/aph.1L093

41. Rodriguez KM. Intrinsic and extrinsic factors affecting patient engagement in diabetes self-management: perspectives of a certified diabetes educator. Clin Ther. 2013;35(2):170-178. doi:10.1016/j.clinthera.2013.01.002

42. Inoue M, Takahashi M, Kai I. Impact of communicative and critical health literacy on understanding of diabetes care and self-efficacy in diabetes management: a cross-sectional study of primary care in Japan. BMC Fam Pract. 2013;14:40. doi:10.1186/1471-2296-14-40

43. Fransen MP, von Wagner C, Essink-Bot ML. Diabetes selfmanagement in patients with low health literacy: ordering findings from literature in a health literacy framework. Patient Educ Couns. 2012;88(1):44-53. doi:10.1016/j.pec.2011.11.015

44. Loke YK, Hinz I, Wang X, Salter C. Systematic review of consistency between adherence to cardiovascular or diabetes medication and health literacy in older adults. Ann Pharmacother. 2012;46(6):863-872. doi:10.1345/aph.1Q718

45. Kalichman SC, Pellowski J, Chen Y. Requesting help to understand medical information among people living with HIV and poor health literacy. AIDS Patient Care STDS. 2013;27(6):326332. doi:10.1089/apc.2013.0056

46. Ownby RL, Waldrop-Valverde D, Caballero J, Jacobs RJ. Baseline medication adherence and response to an electronically delivered health literacy intervention targeting adherence. Neurobehav. HIV Med 2012;4:113-121.

47. Colbert AM, Sereika SM, Erlen JA. Functional health literacy, medication-taking self-efficacy and adherence to antiretroviral therapy. J Adv Nurs. 2013;69(2):295-304. doi:10.1111/j.13652648.2012.06007x

48. Murphy DA, Lam P, Naar-King S, Robert Harris D, Parsons JT, Muenz LR. Health literacy and antiretroviral adherence among HIV-infected adolescents. Patient Education and Counseling. 2010;79(1):25-29. doi:10.1177/0017896912442952

49. Frisch AL, Camerini L, Diviani N, Schulz PJ. Defining and measuring health literacy: How can we profit from other literacy domains? Health Promot Int. 2012;27(1):117-126. doi:10.1093/ heapro/dar043

50. Fineberg H. Health Literacy. Washington, DC:The National Academies Press;2004.

51. McCaffery KJ, Smith SK, Wolf M. The challenge of shared decision making among patients with lower literacy: a framework for research and development. Med Decis Making. 2010;30(1):35-44. doi:10.1177/0272989X09342279

52. Chiang KP, Jackson A. Health literacy and its outcomes: application and extension of elaboration likelihood model. Int J Healthc Manag. 2013;6(3):152-157. doi:10.1179/204797191 3Y.0000000041

53. Clark B. Using law to fight a silent epidemic: the role of health literacy in health care access, quality, and cost. Ann Health Law. 2011;20(2):253-327.

54. HIs-Eu Consortium. Comparative report of health literacy in eight EU member states. the European health literacy survey HLS-EU. Luxembourg:Executive Agency for Health and Consumers;2012.

55. Pleasant A. Health literacy around the world: Part 1. Health literacy efforts outside of the United States. Washington, DC:Institute of Medicine;2012.
56. Parker RM, Ratzen SC, Lurie N. Health Literacy: a policy challenge for advancing high quality health care. Health Aff. 2003;22(4):147.

57. Coulter A. Patient engagement-what works? J Ambul Care Manage. 2012;35(2):80-89. doi:10.1097/JAC.0b013e318249e0fd

58. Edwards M, Wood F, Davies M, Edwards A. The development of health literacy in patients with a long-term health condition: the health literacy pathway model. BMC Public Health. 2012;12:130. doi:10.1186/1471-2458-12-130

59. Coulter A, Ellins J. Effectiveness of strategies for informing, educating, and involving patients. Br Med J. 2007;335(7609):2427. doi:10.1136/bmj.39246.581169.80

60. Nutbeam D. Health Promotion Glossary. Health Promot Int. 1998;13(4):349-364.

61. Baumann L, Ngoc TT. Helping patients with chronic conditions overcome barriers to self-care. Nurse Pract. 2012;37(3):32-38. doi:10.1097/01.NPR.0000411104.12617.64

62. Edwards M, Davies M, Edwards A. What are the external influences on information exchange and shared decision making in healthcare consultations. Patient Educ Couns. 2009;75(1):3752. doi:10.1016/j.pec.2008.09.025

63. Osborn CY, Paasche-Orlow MK, Davis TC, Wolf MS. Health literacy: an overlooked factor in understanding HIV health disparities. Am J Prev Med. 2007;33(5):374-378.

64. Coughlan M, Cronin P, Ryan F. doing a Literature Review in Nursing, Health and Social Care. London:Sage Publication;2013.

65. Hart C. doing a Literature Review: Releasing the Social. London:Sage Publication;1998.

66. Kalichman SC, Cherry C, Kalichman MO, et al. Randomized clinical trial of HIV treatment adherence counseling interventions for people living with HIV and limited health literacy. $J$ Acquir Immune Defic Syndr. 2013;63(1):42-50. doi:10.1097/ QAI.0b013e318286ce49

67. Kalichman SC, Rompa D. Functional Health Literacy Is Associated With Health Status and Health-Related Knowledge in People Living With HIV-AIDS. J Acquir Immune Defic Syndr. 2000;25(4):337-344.

68. Dimmitt Champion J, Harlin B, Collins JL. Sexual risk behavior and STI health literacy among ethnic minority adolescent women. Appl Nurs Res. 2013;26(4):204-209. doi:10.1016/j. apnr.2013.06.003

69. Kalichman SC, Ramachandran B, Catz S. Adherence to combination antiretroviral therapies in HIV patients of low health literacy. J Gen Intern Med. 1999;14(5):267-273. doi:10.1046/ j.1525-1497.1999.00334.x

70. Navarra AM, Neu N, Toussi S, Nelson J, Larson EL. Health literacy and adherence to antiretroviral therapy among HIVinfected youth. J Assoc Nurses AIDS Care. 2014;25(3):203-213. doi:10.1016/j.jana.2012.11.003

71. Kalichman SC, Benotsch E, Suarez T, Catz S, Miller J, Rompa $D$. Health literacy and health-related knowledge among persons living with HIVIAIDS. Am J Prev Med. 2000;18(4):325-331.

72. Howard LM, Tique JA, Gaveta S, Sidat M, Rothman RL, Vermund $\mathrm{SH}$. Health literacy predicts pediatric dosing accuracy for liquid zidovudine. AIDS 2014;28(7):1041-1048. doi:10.1097/ QAD.0000000000000197

73. Zukoski AP, Thorburn S, Stroud J. Seeking information about HIVIAIDS: a qualitative study of health literacy among people living with HIVIAIDS in a low prevalence context. AIDS Care. 2011;23(11):365-375. doi:10.1080/09540121.2011.582077

74. Mackert M, Donovan EE, Mabry A, Guadagno M, Stout PA. Stigma and health literacy: an agenda for advancing research and practice. Am J Health Behav. 2014;38(5):690-698. doi:10.5993/AJHB.38.5.6

75. Kalichman SC, Pope $H$, White D, et al. The association between health literacy and HIV treatment adherence: further evidence from objectively measured medication adherence. J Int Assoc Physicians AIDS Care. 2008;7(6):317-323. doi: $10.1177 / 1545109708328130$ 
76. Osborn CY, Davis TC, Cooper BS, Wolf MS. Health literacy in the context of HIV treatment: introducing the brief estimate of health knowledge and action (BEHKA)-HIV version. AIDS Behav. 2010;14(1):181-188. doi:10.1007/s10461-008-9484

77. Waldrop-Valverde D, Jones DL, Jayaweera D, Gonzalez P, Romero J, Ownby RL. Gender differences in medication management capacity in HIV infection: the role of health literacy and numeracy. AIDS Behav. 2009;13(1):46-52. doi:10.1007/ s10461-008-9425-x

78. Hicks G, Barragan M, Franco-Paredes C, Williams MV, del Rio C. Health literacy is a predictor of HIVIAIDS knowledge. Fam Med. 2006;38(10):717-723.

79. Graham J, Bennett IM, Holmes WC, Gross R. Medication beliefs as mediators of the health literacy - Antiretroviral adherence relationship in HIV-infected individuals. AIDS Behav. 2007;11(3):385-392.

80. Wolf MS, Davis TC, Cross JT, Marin E, Green K, Bennett CL. Health literacy and patient knowledge in a Southern US HIV clinic. Int J STD AIDS. 2004;15(11):747-752.

81. Mooss A, Brock-Getz P, Ladner R, Fiaño T. The relationship between health literacy, knowledge of health status, and beliefs about HIVIAIDS transmission among Ryan White clients in Miami. Health Educ J. 2013;72(3):292-299.

82. van Servellen G, Carpio F, Lopez M, et al. Program to enhance health literacy and treatment adherence in low-income HIVinfected Latino men and women. AIDS Patient Care STDS. 2003;17(11):581-594.

83. Laws MB, Danielewicz M, Rana A, Kogelman L, Wilson IB. Health literacy in Hiv treatment: accurate understanding of key biological treatment principles is not required for good Art adherence. AIDS Behav. 2015;19(4):635-644. doi:10.1007/ s10461-014-0931-8

84. Valenti WM. Health literacy, HIV and outcomes. AIDS Reader. 2007;17(3):124-126.

85. Ownby RL, Waldrop-Valverde D, Hardigan P, Caballero J, Jacobs $R$, Acevedo A. Development and validation of a brief computeradministered HIV-related health literacy scale (HIV-HL). AIDS Behav. 2013;17(2):710-718. doi:10.1007/s10461-012-0301-3

86. Ohl M, Harris A, Nurudtinova D, Cai X, Drohobyczer D, Overton ET. Do brief screening questions or provider perception accurately identify persons with low health literacy in the HIV primary care setting? AIDS Patient Care STDS. 2010;24(10):623-629. doi:10.1089/apc.2009.0319

87. Nachega JB, Morroni C, Zuniga JM, et al. HIV treatment adherence, patient health literacy, and health care providerpatient communication: results from the 2010 AIDS treatment for life international survey. $J$ Int Assoc Physicians AIDS Care. 2012;11(2):128-133. doi:10.1177/1545109712437244

88. Wawrzyniak AJ, Ownby RL, McCoy K, Waldrop-Valverde D. Health literacy: impact on the health of HIV-infected individuals. Curr HIVIAIDS Rep. 2013;10(4):295-304. doi:10.1007/s11904013-0178-4

89. Barragán M, Hicks G, Williams MV, Franco-Paredes C, Duffus W, del Rio C. Low health literacy is associated with HIV test acceptance. J Gen Intern Med. 2005;20(5):422-425. doi:10.1111/

\section{j.1525-1497.2005.40128.x}

90. Paasche-Orlow MK, Cheng DM, Palepu A, Meli S, Faber V, Samet JH. Health literacy, antiretroviral adherence, and HIVRNA suppression: a longitudinal perspective. J Gen Intern Med. 2006;21(8):835-840.

91. Nokes KM, Coleman CL, Cashen M, Dole P, Sefcik E, Hamilton MJ. Health literacy and health outcomes in HIV seropositive persons. Res Nurs Health. 2008;30(6):620-627.

92. Koskan A, Friedman DB, Hilfinger Messias DK. Health literacy among hispanics: A systematic research review (1992-2008). Hispanic Health Care Int. 2010;8(2):65-76.

93. van Servellen G, Brown JS, Lombardi E, Herrera G. Health literacy in low-income Latino men and women receiving antiretroviral therapy in community-based treatment centers. AIDS Patient Care STDS. 2003;17(6):283-298.

94. van Servellen G, Nyamathi A, Carpio F, et al. Effects of a treatment adherence enhancement program on health literacy, patient-provider relationships, and adherence to HAART among low-income HIV-positive Spanish-speaking Latinos. AIDS Patient Care STDS. 2005;19(11):745-759.

95. Bynum SA, Wigfall LT, Brandt HM, Richter DL, Glover SH, Hébert JR. Assessing the influence of health literacy on HIV-positive women's cervical cancer prevention knowledge and behaviors. J Cancer Educ. 2013;28(2):352-356. doi:10.1007/s13187-0130470-4

96. Rikard RV, Thompson MS, Head R, McNeil C, White C. Problem posing and cultural tailoring: developing an HIV/ AIDS health literacy toolkit with the African American Community. Health Promot Pract. 2012;13(5):626-636. doi: $10.1177 / 1524839911416649$

97. McMichael C, Gifford S. It is good to know now...before it's too late: promoting sexual health literacy amongst resettled young people with refugee backgrounds. Sex Cult. 2009;13(4):218236. doi:10.1007/s12119-009-9055-0

98. Drainoni ML, Rajabiun S, Rumptz M, et al. Health literacy of HIVpositive individuals enrolled in an outreach intervention: Results of a cross-site analysis. J Health Commun. 2008;3(3):287-302. doi:10.1080/10810730801985442

99. Car J, Lang B, Colledge A, Ung C, Majeed A. Interventions for enhancing consumers' online health literacy. Cochrane Database Syst Rev. 2011;6:CD007092. doi:10.1002/14651858. CD007092.pub2

100. Robinson C, Graham J. Perceived Internet health literacy of HIVpositive people through the provision of a computer and Internet health education intervention. Health Info Libr J. 2010;27(4):295303. doi:10.1111/j.1471-1842.2010.00898.x

101. Pomerantz KL, Muhammad AA, Downey S, Kind T. Connecting for health literacy: health information partners. Health Promot Pract. 2010;11(1):79-88. doi:10.1177/1524839908318166

102. Rivero-Méndez M, Suárez E, Solís-Báez SS, et al. Internal consistency of the spanish health literacy test (TOFHLA-SPR) for Puerto Rico. Puerto Rico Health Sci J. 2010;29(1):49-53.

103. Kickbusch IS. Health literacy: addressing the health and education divide. Health Promot Int. 2001;16(3):289-297. doi:10.1093/heapro/16.3.289 